Journal of Sustainable Development of Transport and Logistics

journal home page: http://jsdtl.sciview.net

\title{
Solving the problem of elasticity for round thick plates at axially symmetric strain
}

\section{Oleksiy Hvertsev *, Ninel Selivanova **}

* National Transport University,

1, M. Pavlenko-Omelianovych str., Kyiv 01010, Ukraine

** National Transport University,

1, M. Pavlenko-Omelianovych str., Kyiv 01010, Ukraine

e-mail:nel_s@bk.ru

Senior Lecturer, Department of Transport Law and Logistics

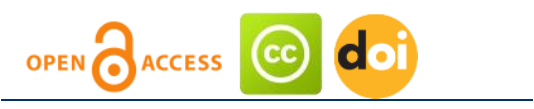

\section{Article history:}

Received: October, 2016

1st Revision: October, 2016

Accepted: November, 2016

\section{DOI:}

10.14254/jsdtl.2016.1-1.7

\begin{abstract}
An exact solution of the equations of elasticity for round plates loaded axially symmetric. The problem of bending round plates, which are under the influence of normal forces attached to any law to load any type of resistance. It is shown that pasture circular plate under axially symmetric load leads to appearance of temperature field.
\end{abstract}

Keywords: theory of elasticity, axisymmetric function movement, temperature change, thermodynamically reversible (inverse) process, bending round plate. 


\section{Main material}

It is known (Holdenblat, I.I.,1969; Popovych, P. V., \& Slobodyan, Z. B., 2014) that the exact solution of elasticity considered only solid round plates, loaded evenly distributed pressure constant intensity. The law can be an arbitrary load type and resistance plate type.

Differential equations of equilibrium in cylindrical coordinates are following (Holdenblat, I.I.,1969):

$$
\Delta u_{1}-\frac{u_{1}}{r^{2}}+\frac{e,_{1}}{1-2 v}-2 \frac{(1+v)}{(1-2 v)} \alpha \theta,_{1}=0 ; \Delta u_{3}+\frac{e_{3}}{1-2 v}-2 \frac{(1+v)}{(1-2 v)} \alpha \theta,_{3}=0
$$

and stresses:

$$
\sigma_{11,1}+\sigma_{13,1}+\frac{\sigma_{11}-\sigma_{22}}{r}=0 ; \quad \sigma_{13,1}+\sigma_{33,3}+\frac{1}{r} \sigma_{13}=0
$$

In equations (1) and (2) the index after comma places means of partial corresponding coordinate $r$ or $z$; $u_{1}$ and $u_{3}$ components respectively radial and axial movement; $\Delta u$ - Laplace operator of movement $u_{i}(i=1,3)$; $u_{i}(i=1,3) ; \sigma_{11}, \sigma_{12}, \sigma_{13}$ - under radial components, county, axial and shear stresses.

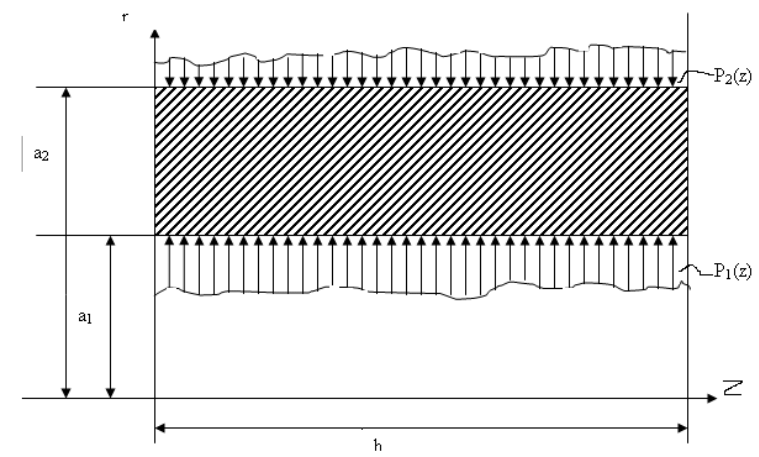

Fig. 1. Scheme plate under uniformly distributed load

Components of stress are determined by Hook`s law (Germain, P., 1983).

$$
\sigma_{i j}=2 G\left(e_{i j}+\frac{v}{1-2 v} e \delta_{i j}-\frac{1+v}{1-v} \alpha \theta \delta_{i j}\right), i, j=1,2,3
$$

under certain dependencies between deformations and displacements:

$$
e_{11}=u_{1,1} ; e_{22}=\frac{1}{r} u_{1} ; e_{33}=u_{3,1} ; 2 e_{13}=u_{1,3}+u_{3,1}
$$

Here $\delta_{i j}$ - Kronecker's symbol; and $\alpha$ and $v$ - coefficients of linear thermal expansion and Poisson; $e=e_{11}+e_{22}+e_{33}-$ Thermal expansion; $G=\frac{E}{2(1+v)}-$ Shear modulus; $E$ - Modulus of elasticity.

For circuit system equilibrium equations (1) should be added heat equation (Germain, P., 1983):

$$
\Delta \theta+\frac{1}{\lambda} W=0
$$

By $W$ marked quantity of heat produced or absorbed per unit volume of the body with its deformation; $\lambda$ - Thermal conductivity; $\Delta \theta=\theta,_{11}+\frac{1}{r} \theta,_{11}+\theta,_{33}$.

Solving the problem of bending circular plates under arbitrary axisymmetric load intensity $P(r)_{x}$ requires the setting of normal axial tension $\sigma_{33}$. 
For its definition use the method based on translational approximations solution proposed by one of the authors. After mathematical transformations we get value of $\sigma_{33}$ :

$$
\sigma_{33}=-P(r) \frac{12}{h^{3}}\left(\frac{z^{3}}{6}-\frac{z h^{2}}{8}-\frac{h^{3}}{24}\right)
$$

That will satisfy the following conditions:

$$
\sigma_{33}=-P(r), \text { при } z=-\frac{h}{2} \text { і } \sigma_{33}=0, \text { при } z=\frac{h^{2}}{2}
$$

Where $h$ - Thickness circular plate.

Knowing the tension (6) turn to the solving of thick plates bending problem, using function $\phi_{(r, z)}$. For more accurate solution to this problem should be considered polytropic, h.e. not isothermal and not adiabatic, thermodynamic process. Function $\psi_{(r, z)}$ linked to thermostatic potential displacement formula (Germain, P., 1983):

$$
\phi_{(r, z)}=\frac{1+v}{1-x} \psi(r, z)
$$

In this case, we present the movement as such:

$$
u_{1}(r, z)=\frac{1+v}{1-v} \psi_{, 1} ; u_{3}(r, z)=\frac{1+v}{1-v} \psi_{, 3}
$$

thermal expansion $e$ we find in expression (4) given (9):

$$
e=e_{11}+e_{22}+e_{33}=\frac{1+v}{1-v}\left[\psi_{, 11}+\frac{1}{r} \psi_{, 1}+\psi_{, 33}\right]=\frac{1+v}{1-v} \Delta \psi
$$

Substituting movement (9) and volume expansion (10) in equation (1) we get:

$$
\begin{aligned}
& \frac{2(1+v)}{1-v}(\Delta \psi)_{, 1}-\frac{2(1+v)}{1-v} 2 \theta_{, 1}=0 \\
& \frac{2(1+v)}{1-v}(\Delta \psi)_{, 3}-\frac{2(1+v)}{1-v} 2 \theta_{, 3}=0
\end{aligned}
$$

Integrating the first equation in $r$ and the second on $z$, we obtain the formula for determining the temperature variable:

$$
2 \theta=\Delta \psi
$$

Suppler find out Hooke's law (Landau, L.D. \& Livshits, E.M., 1987), where the temperature change $\theta$ can be determined using equation (12).

Then we have:

$$
\begin{gathered}
\sigma_{13}=\frac{E}{1-v} \psi_{, 13} ; \sigma_{33}=-\frac{E}{1-v} \frac{1}{r}\left(r \psi_{, 1}\right)_{, 1} ; \\
\sigma_{11}=-\frac{E}{1-v}\left(\frac{1}{r} \psi_{, 1}+\psi_{, 3}\right) ; \sigma_{22}=-\frac{E}{1-v}\left(\psi_{, 11}+\psi_{, 33}\right) ;
\end{gathered}
$$


To solve the equations of elasticity should select a function of displacement $\psi(r, z)$ in a way that allows you to satisfy specific boundary conditions of the problem.

Next, consider bending round plates in the exact formulation of the problem of elasticity, which is attached under normal force $P(r)$ any law load symmetrically about the axis of rotation $z$, when any type of resistance.

Using strain of $\sigma_{33}$ from (6) that satisfies the conditions (7) and compared with the voltage $\sigma_{33}$ from the equation (13), we obtain the differential equation for determining the function $\psi(r, z)$.

$$
\left(r \psi_{, 1}\right)_{, 1}=\frac{1-v}{E} \frac{12}{h^{3}}\left(\frac{z^{3}}{6}-\frac{z h^{2}}{8}+\frac{h^{3}}{24}\right) r P(r)
$$

Integrating this equation by $r$ between $a_{1}$ to $r$, We get:

$$
\psi_{, 1}=\frac{1-v}{E} \frac{12}{h^{3}}\left(\frac{z^{3}}{6}-\frac{z h^{2}}{8}+\frac{h^{3}}{24}\right) \frac{1}{r} \int_{a_{1}}^{r} r P(r) d r+\frac{1}{r} f_{1}(z)
$$

Where $f_{1}(z)$ - Arbitrary function integration; $a_{1}$ - The radius of the central aperture plate.

If the solid plate $\left(a_{1}=0\right)$, Then $f_{1}(z)$ it should be considered to be zero for finite displacements $u(r)$.

Repeating the process of integrating with $r$ between $a_{1}$ to $r$, We get:

$$
\psi_{(r, z)}=\frac{1-v}{E} \frac{12}{h^{3}}\left(\frac{z^{3}}{6}-\frac{z h^{2}}{8}+\frac{h^{3}}{24}\right) \times \int_{a_{1}}^{r} \frac{1}{r} \int_{a_{1}}^{r} r P(r) d r d r+f_{1}(z) \ln \frac{r}{a}+f_{2}(z)
$$

Where $f_{2}(z)$ - arbitrary function integration.

For ease of calculation, consider a solid cylindrical plate $\left[a_{1}=0 ; f_{1}(z)=0\right]$. Then:

$$
\psi_{(r, z)}=\frac{1-v}{E} \frac{12}{h^{3}}\left(\frac{z^{3}}{6}-\frac{z h^{2}}{8}+\frac{h^{3}}{24}\right) \int_{0}^{r} \frac{1}{r} \int_{0}^{r} r P(r) d r d r+f_{2}(z)
$$

Differentiating function (17) to $r$ and $z$, We get:

$$
\psi_{, 13}=\frac{1-v}{E} \frac{12}{h^{3}}\left(\frac{z^{2}}{6}-\frac{h^{2}}{8}\right) \frac{1}{r} \int_{0}^{r} r P(r) d r
$$

Putting $\psi_{, 13}$ in tension $\sigma_{13}$ the equations (13) gives:

$$
\sigma_{13}=\frac{6}{h^{3}}\left(z^{2}-\frac{h^{2}}{4}\right) \frac{1}{r} \int_{0}^{r} r P(r) d r
$$

Boundary conditions for shear stresses (19) carried integrally on the edge of a circular plate, it means that at the $\sigma_{13}$ performance boundary conditions on average requires voltage was set on the edge of the plate to the support reactions:

$$
A=\left[\int_{-\frac{h}{2}}^{\frac{h}{2}} \sigma_{13} d z\right]_{r=a}=-\frac{1}{a} \int_{0}^{a} r P(r) d r
$$


Thus, the tangential stress (19) exactly satisfy the boundary conditions on planes $z= \pm \frac{h}{2}$ and integrally on the edge of the cylindrical surface.

Substituting the function (17) in the formula (13) for normal radial stress $\sigma_{11}$ and finding function $f_{2}(z)$ with boundary conditions $\sigma_{11}=0$ at $r=a$, We get:

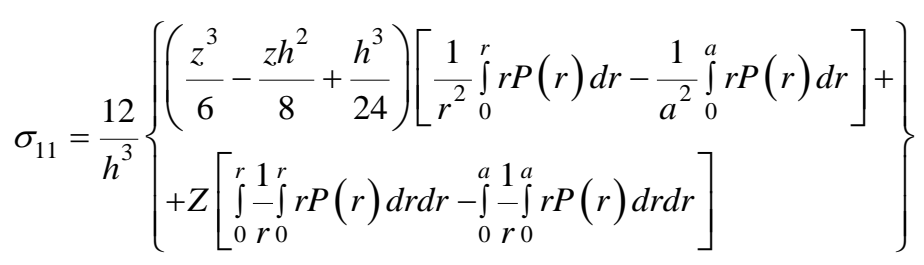

For the district voltage $\sigma_{22}$ from the equations (13) we get:

$$
\sigma_{22}=-\frac{12}{h^{3}}\left\{\begin{array}{r}
\left(\frac{z^{3}}{6}-\frac{z h^{2}}{8}+\frac{h^{3}}{24}\right) \times \times\left[P(r)-\left(\frac{1}{r^{2}} \int_{0}^{r} r P(r) d r-\frac{1}{a^{2}} \int_{0}^{a} r P(r) d r\right)\right]+ \\
+z\left[\int_{0}^{r} \frac{1}{r} \int_{0}^{r} r P(r) d r d r-\int_{0}^{a}-\int_{0}^{a} r P(r) d r d r\right]
\end{array}\right\}
$$

The resulting voltage satisfying balance equation (2) and boundary conditions: $\sigma_{33}=-P(r)$, at $z=-\frac{h}{2}$; $\sigma_{33}=0$ at $z=\frac{h}{2} ; \sigma_{13}=0$ at $z= \pm \frac{h}{2}$; and integrated on the appliance: $\sigma_{11}=0$ at $r=a$.

This is the first time when we get this kind of solving of the bending thick plate problem. Terms of impedance (resistance) plates allow you to determine the exact value movements on formulas (8), using (17).

Moving $u_{1}$ and $u_{2}$ accepted as such:

$$
\begin{gathered}
u_{1}(r, z)=\frac{1+V}{1-V}\left[\psi_{, 1}+\frac{1}{r}\left(A_{5} z-A_{3}\right)\right]+r\left(A_{4} z+A_{6} \frac{1}{2}\right) \\
u_{3}(r, z)=\frac{1+V}{1-V}\left[\psi_{, 3}+A_{5} \ln r\right]-A_{4}\left(\frac{V}{1-V} z^{2}+\frac{r^{2}}{2}\right)-\frac{V}{1-V} z A_{6}+A_{7},
\end{gathered}
$$

where $A_{i}$ - Arbitrary constants of integration.

By movements (23) we find Voltage:

$$
\begin{gathered}
\sigma_{13}=\frac{E}{1-V} \psi_{, 13} ; \sigma_{33}=-\frac{E}{1-V} \frac{1}{r}\left(r \psi_{, 1}\right)_{, 1} \\
\sigma_{11}=\frac{E}{1-V}\left[-\frac{1}{r} \psi_{, 1}-\psi_{, 33}-\frac{1}{r^{2}}\left(A_{5} z-A_{3}\right)+A_{4} z+A_{6} \frac{1}{2}\right] \\
\sigma_{22}=\frac{E}{1-V}\left[-\psi_{, 11}-\psi_{, 33}+\frac{1}{r^{2}}\left(A_{5} z-A_{3}\right)+A_{4} z+A_{6} \frac{1}{2}\right]
\end{gathered}
$$

Expressions (23) and (24) is the exact solution of equilibrium equations (1) and (2) because the last turn after substituting for identity. Function $\psi(r, z)$ must satisfy the equation (12), by which is possible to determine the temperature variable $\theta(r, z)$, which is forming because of the actions of external pressures and dependence on boundary conditions. 
To illustrate the elastic and thermodynamic reversibility above mentioned problems of elasticity theory for axisymmetric deformation of plates, we simplify decision, $P(r)=P_{0}=$ const suggesting that see Fig. 1

Then we have the function of displacement (17):

$$
\psi(r, z)=\frac{1-V}{E} \frac{12}{h^{3}} P_{0}\left(\frac{z^{3}}{6}-\frac{z h^{2}}{8}+\frac{h^{3}}{24}\right) \frac{r^{2}}{4}+f_{2}(z)
$$

For the tangent of tension (19), choose:

$$
\sigma_{13}=\frac{3}{h^{3}} P_{0}\left(z^{3}-\frac{h^{2}}{4}\right) r
$$

The size of the support reactions on the edge of the plate is:

$$
A=-\frac{a}{2} P_{0}
$$

Radial (21) and county (22) voltage match:

$$
\sigma_{11}=\sigma_{22}=\frac{3 z}{h^{3}} P_{0}\left(a^{2}-r^{2}\right)
$$

For functions $f_{2}(z)$ ends

$$
f_{2}(z)=\frac{1-V}{E} \frac{6}{h^{3}} P_{0}\left(\frac{z^{5}}{120}-\frac{z^{3} h^{2}}{48}+\frac{z^{2} h^{3}}{48}+\frac{z^{3} a}{12}\right)+C_{1} z+C_{2}
$$

where $C_{1}$ and $C_{2}$ - Arbitrary constants of integration.

Find displacement (9):

$$
\begin{gathered}
u_{1}(r, z)=\frac{1+V}{1-V} \psi_{, 1}=\frac{1+V}{E} \frac{12}{h^{3}} P_{0}\left(\frac{z^{3}}{6}-\frac{z^{8} h^{2}}{8}+\frac{h^{3}}{24}\right) \frac{r}{2} \\
u_{3}(r, z)=\frac{1+V}{1-V} \psi_{, 3}=\frac{1+V}{E} \frac{3}{2 h^{3}} P_{0} \times\left[r^{2}\left(z^{2}-\frac{h^{2}}{4}\right)-\left(\frac{z^{4}}{6}-\frac{h^{2} z^{2}}{4}+\frac{z h^{3}}{6}+z^{2} a^{2}\right)\right]+\frac{1+V}{1-V} C_{1}
\end{gathered}
$$
the axis.

Radial displacement (28) does such resistance plate, allowing the movement of plates in the direction of Normal movement (30) allows for different anchor point to secure the appliance on their vertical displacement.

Consider as an example hinge fixing points circuit boards in the median plane, that is:

$$
u_{3}=0 ; r=a ; z=0
$$

Satisfying these boundary conditions for moving (31), we obtain for any sustainable integration $C_{1}$ :

$$
\frac{1+V}{1-V} C_{1}=\frac{1+V}{E} \frac{3}{2 h^{3}} P_{0} \frac{a^{2} h^{2}}{4}
$$


After substituting this value in (30), we get:

$$
u_{3}(r, z)=\frac{1+V}{E} \frac{3}{2 h^{3}} P_{0} \times\left[r^{2}\left(z^{2}-\frac{h^{2}}{4}\right)+\frac{a^{2} h^{2}}{4}-\left(\frac{z^{4}}{6}-\frac{z^{2} h^{2}}{4}+\frac{z h^{3}}{6}+a^{2} z^{2}\right)\right]
$$

Voltage corresponding displacement (30) and (33) are as follows:

$$
\begin{gathered}
\sigma_{33}=-\frac{6}{h^{3}} P_{0}\left(\frac{z^{3}}{3}-\frac{h^{2} z}{4}+\frac{h^{3}}{12}\right) ; \\
\sigma_{13}=\frac{3}{h^{3}} P_{0}\left(z^{2}-\frac{h^{2}}{4}\right) r ; \\
\sigma_{11}=\sigma_{22}=\frac{3}{h^{3}} P_{0}\left(a^{2}-r^{2}\right) z
\end{gathered}
$$

The function of displacement (25), after substituting (29) is:

$$
\psi(r, z)=\frac{1-V}{E} \frac{6}{h^{3}} P_{0} \times\left[\left(\frac{z^{3}}{6}-\frac{z h^{2}}{8}+\frac{h^{3}}{24}\right) \frac{r^{2}}{4}-\left(\frac{z^{5}}{120}-\frac{z^{3} h^{2}}{48}+\frac{z^{2} h^{3}}{48}+\frac{a^{2} z^{2}}{12}\right)+\frac{a^{2} z^{2}}{16} z\right]+C_{2}
$$

Where $C_{2}$ - Arbitrary constants of integration.

Finding the temperature variable $\theta(r, z)$ in cylindrical circular plate, which arises from the action of external load $P_{0}$.

$$
\alpha \theta=\Delta \psi=\psi_{, 11}+\frac{1}{r} \psi_{, 1}+\psi_{, 33}
$$

Substituting the function (35) to (36), we find:

$$
\theta(r, z)=\frac{(1-V)}{E \alpha} \frac{6}{h^{3}} P_{0}\left[\frac{z^{3}}{6}-\frac{z h^{2}}{8}+\frac{h^{3}}{24}-\left(a^{2}-r^{2}\right) \frac{z}{2}\right]
$$

We know that the external load, surface and volume, leading to deformation of the body while causing the appearance of temperature changes in it (Landau, L.D. \& Livshits, E.M., 1987). The change in temperature makes $\theta=T-T_{0}$ where $T$ - Absolute temperature points of the body, $T_{0}$ - Temperature fatigue-free body at the time $t=0$.

Thus, when changing deformation point temperature of the body is a result of absorption or heat insulated elastic body, with its interaction of the environment (Melanie, E., \& Parkus, G., 1958). This temperature change curved round to the load plate $P_{0}$ defines dependence (37).

If you remove the load $P_{0}=0$ voltage (34) and temperature change (37) disappear and the plate returned to its not intense and deformed original condition. The process is very slow deformation, which means it will be thermodynamically reversible (Timoshenko, S.P., \& Huder, J., 1979).

Exploring plate deformation and temperature change it from outside the load. Substituting axial displacement (33) $z=-\frac{h}{2}$ we get the upper layers of compression plates in key areas:

$$
u_{3}\left(r_{1}-\frac{h}{2}\right)=\frac{1+V}{E} \frac{3 h}{64} P_{0}>0
$$

For the bottom surface of the plate at $z=+\frac{h}{2}$ we get: 


$$
u_{3}\left(r_{1} \frac{h}{2}\right)=-\frac{1+V}{E} \frac{3 h}{64} P_{0}<0,
$$

that will have stretched zone.

It is a known fact from the theory of physical structures bend. Then examine temperature change of formula (33) for a compressed zone. Find:

$$
\begin{gathered}
\theta\left(r_{1}-\frac{h}{2}\right)=\frac{1-V}{E \alpha} \frac{3}{h^{2}} P_{0}\left(\frac{h^{2}}{3}+a^{2}-r^{2}\right) \geq 0, \text { або } \\
T\left(r_{1}-\frac{h}{2}\right)=T_{0}+\frac{1-V}{E \alpha} \frac{3}{h^{2}} P_{0}\left(\frac{h^{2}}{3}+a^{2}-r^{2}\right) .(r \leq a)
\end{gathered}
$$

To get stretched zone:

$$
\begin{gathered}
\theta\left(r_{1} \frac{h}{2}\right)=-\frac{1-V}{E \alpha} \frac{3}{h^{2}} P_{0}\left(a^{2}-r^{2}\right) \leq 0, \text { aбо } \\
T\left(r_{1} \frac{h}{2}\right)=T_{0}-\frac{1-V}{E \alpha} \frac{3}{h^{2}} P_{0}\left(a^{2}-r^{2}\right) . \\
(r \leq a)
\end{gathered}
$$

As you can see, the temperature of the compressed zone will be slightly higher then the temperature of stretched zone. In other words, compressed zone generates heat $\left(T>T_{0}\right)$ and stretched it absorbs zone $\left(T<T_{0}\right)$, That is a plate heat exchange with the environment. A similar theory was developed by Karl Zener (Feng, J., 1986) for thin plate bending vibrations at her and was brilliant experimental confirmation.

Substituting variable temperature (37), which appears by bending a round plate of uniformly distributed load intensity constant in the equation (5) is the heat capacity flow in areas of the plate.

$$
W=-\lambda \Delta \theta=-\lambda \frac{(1-V)}{E \alpha} \frac{18 z}{h^{3}} P_{0}
$$

For compressed zone at the plate $z=-\frac{h}{2}$ :

$$
W=\lambda \frac{(1-V)}{E \alpha} \frac{9}{h^{2}} P_{0}>0
$$

the heat goes.

To straighten the zone plate $z=\frac{h}{2}$ :

$$
W=-\lambda \frac{(1-V)}{E \alpha} \frac{9}{h^{2}} P_{0}<0
$$

goes absorb heat from the environment.

Consequently, there is heat conduction through the heat flow from the compressed zone to straighten.

\section{Conclusions}

Thus, in solving the problem of elasticity theory for axisymmetric bending loaded circular plates, the formula of intensity and displacements received for the first time. Law of loading can be any type, and the type of resist - either. The resulting solution indicates that the effect of external forces leads to the appearance of temperature changes at bending of round plates and occurrence of heat flow. 


\section{Appendix A. Supplementary material}

Supplementary data associated with this article can be found, in the online version, at http://jsdtl.sciview.net

\section{Funding}

The authors received no direct funding for this research.

\section{Citation information}

Hvertsev, O., \& Selivanova, N. (2016). Solving the problem of elasticity for round thick plates at axially symmetric strain. Journal of Sustainable Development of Transport and Logistics, 1(1), 40-48. doi: 10.14254/jsdtl.2016.1-1.7.

\section{References}

Feng, J. (1986). Machines, energy, entropy. Moscow, USSR: Mir. (in Russian).

Germain, P. (1983). Kurs mekhaniki sploshnykh sred [The course of continuum mechanics]. Moscow, USSR: Higher School. (in Russian).

Holdenblat, I.I. (1969). Nelineynaya teoriya problem uprugosti [Nonlinear theory upruhosty problems]. Moscow, USSR: Nauka. (in Russian).

Landau, L.D. \& Livshits, E.M. (1987). Teoriya uprogosti [Theory of elasticity]. Moscow, USSR: Science, VII. (in Russian).

Melanie, E., \& Parkus, G. (1958). Termouprugie napryazheniya, vyzyvaemye statsionarnymi temperaturnymi polyami [Thermoelastic stresses caused by stationary temperature fields]. Moscow, USSR: Fyzmathyz. (in Russian).

Popovych, P. V., \& Slobodyan, Z. B. (2014). Corrosion and Electrochemical Behaviors of 20 Steel and St.3 Steel in Ammonium Sulfate and Nitrophoska. Materials Science, 49(6), 819-826. (in Russian).

Timoshenko, S.P., \& Huder, J. (1979). Teoriya uprugosti [Theory of elasticity]. Moscow, USSR: Nauka. (in Russian).

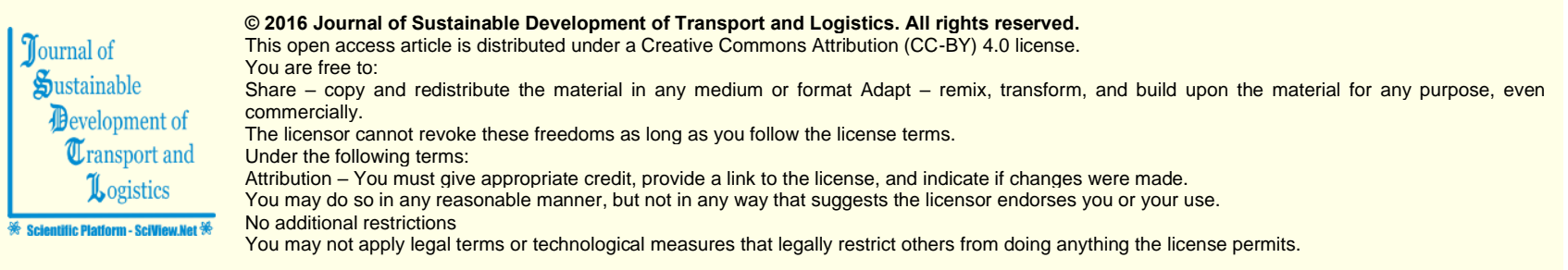

Journal of Sustainable Development of Transport and Logistics (ISSN: 2520-2979) is published by Scientific Publishing House "CSR", Poland, EU and Scientific Publishing House "SciView", Ukraine

Publishing with JSDTL ensures:

- Immediate, universal access to your article on publication

- High visibility and discoverability via the JSDTL website

- Rapid publication

- Rapid publication

- Guaranteed legacy preservation of your article

Submit your manuscript to a JSDTL at http://jsdtl.sciview.net/ or submit.jsdtl@sciview.net 